\title{
Functional Condensation Polymers
}




\title{
Functional Condensation Polymers
}

\author{
Edited by \\ Charles E. Carraher, Jr. \\ Florida Atlantic University \\ Boca Raton, Florida and \\ Florida Centerfor Environmental Studies \\ Palm Beach Gardens, Florida

\section{Graham G. Swift} \\ G.S.P.C., Inc. \\ Chapel Hill, North Carolina
}


eBook ISBN: $\quad 0-306-47563-4$

Print ISBN: $\quad$ 0-306-47245-7

C2002 Kluwer Academic Publishers

New York, Boston, Dordrecht, London, Moscow

Print @2002 Kluwer Academic/Plenum Publishers

New York

All rights reserved

No part of this eBook may be reproduced or transmitted in any form or by any means, electronic, mechanical, recording, or otherwise, without written consent from the Publisher

Created in the United States of America

Visit Kluwer Online at:

http://kluweronline.com

and Kluwer's eBookstore at:

http://ebooks. kluweronline.com 


\section{Contributors}

Kumudi Abey, Florida Atlantic University, Boca Raton, Florida

Stephen Andrasik, University of Central Florida, Orlando, Florida

R. Scott Armentrout, Eastman Chemical Company, Kingsport, Tennessee

Grant D. Barber, University of Southern Mississippi, Hattiesburg, Mississippi

T. Beck, Pharmacia Corporation, Chesterfield, Missouri

Kevin D. Belfield, University of Central Florida, Orlando, Florida

Carl E. Bonner, Norfolk State University, Norfolk, Virginia

K. Botwin, Pharmacia Corporation, Chesterfield, Missouri

Timothy L. Boykin, Bayer Corporation, Pittsburgh, Pennsylvania

Charles E. Carraher, Jr., Florida Atlantic University, Boca Raton, Florida and Florida Center for Environmental Studies, Palm Beach Gardens, Florida

Shawn M. Carraher, Texas A\&M University, Commerce, Texas

Donna M. Chamely, Florida Atlantic University, Boca Raton, Florida

Victor M. Chapela, Beremerita Universidad Autonoma de Puebla, Puebla, Mexico

David M. Collard, Georgia Institute of Technology, Atlanta, Georgia

Ann-Marie Francis, Florida Atlantic University, Boca Raton, Florida

Holger Frey, Albert-Ludwigs Universität, Freiburg, Germany

Sakuntala Chatterjee Ganguly, Indian Institute of Technology, Kharagpur, India and SAKCHEM, Mowbray, Tasmania, Australia

Jerome E. Haky, Florida Atlantic University, Boca Raton, Florida

Shiro Hamamoto, Toyobo Research Center Company, Ohtsu, Japan

Mason K. Harrup, Idaho National Engineering and Environmental Laboratory, Idaho Falls, Idaho

James Helmy, Florida Atlantic University, Boca Raton, Florida

Samuel J. Huang, University of Connecticut, Storrs, Connecticut 
R. Jansson, Pharmacia Corporation, Chesterfield, Missouri

Michael G. Jones, Idaho National Engineering and Environmental Laboratory, Idaho Falls, Idaho

Huaiying Kang, Virginia Polytechnic Institute and State University, Blacksburg, Virginia

Kota Kitamura, Toyobo Research Center Company, Ohtsu, Japan

D. Kunneman, Pharmacia Corporation, Chesterfield, Missouri

G. Lange, Pharmacia Corporation, Chesterfield, Missouri

Wesley W. Learned, Flying L Ranch, Billings, Oklahoma

Stephen C. Lee, Pharmacia Corporation, Chesterfield, Missouri and Department of Chemical Engineering and the Biomedical Engineering Center, Ohio State University, Columbus, Ohio

Timothy E. Long, Virginia Polytechnic Institute and State University, Blacksburg, Virginia

Shahin Maaref, Norfolk State University, Norfolk, Virginia

Joseph M. Mabry, University of Southern California, Los Angeles, California

T. Miller, Pharmacia Corporation, Chesterfield, Missouri

Robert B. Moore, University of Southern Mississippi, Hattiesburg, Mississippi

Alma R. Morales, University of Central Florida, Orlando, Florida

Rolf Mulhaupt, Albert-Ludwigs Universität, Freiburg, Germany

David Nagy, Florida Atlantic University, Boca Raton, Florida

Junko Nakao, Toyobo Research Center Company, Ohtsu, Japan

Rei Nishio, Teijin Ltd., Iwakuni, Yamaguchi, Japan

R. Parthasarathy, Pharmacia Corporation, Chesterfield, Missouri

Zhonghua Peng, University of Missouri-Kansas City, Kansas City Missouri

Judith Percino, Benemerita Universidad Autonoma de Puebla, Puebla, Mexico

Fred Pflueger, Florida Atlantic University, Boca Raton, Florida

Dirk Poppe, Albert-Ludwigs Universität, Freiburg, Germany

Monica Ramos, University of Connecticut, Storrs, Connecticut

Alberto Rivalta, Florida Atlantic University, Boca Raton, Florida

John R. Ross, Florida Atlantic University, Boca Raton, Florida 
E. Rowold, Pharmacia Corporation, Boca Raton, Florida

Jiro Sadanobu, Teijin Ltd., Iwakuni, Yamaguchi, Japan

Yoshimitsu Sakaguchi, Toyobo Research Center Company, Ohtsu, Japan

Alicia R. Salamone, Florida Atlantic University, Boca Raton, Florida

Katherine J. Schafer, University of Central Florida, Orlando, Florida

David A. Schiraldi, Next Generation Polymer Research, Spartanburg, South Carolina

Jianmin Shi, Eastman Kodak, Rochester, New York

Deborah W. Siegmann-Louda, Florida Atlantic University, Boca Raton, Florida

Robin E. Southward, College of William and Mary, Williamsburg, Virginia

Herbert Stewart, Florida Atlantic University, Boca Raton, Florida

Sam-Shajing Sun, Norfolk State University, Norfolk, Virginia

Hiroshi Tachimori, Toyoba Research Center Company, Ohtsu, Japan

Satoshi Takase, Toyoba Research Center Company, Ohtsu, Japan

D. Scott Thompson, College of William and Mary, Williamsburg, Virginia

D. W. Thompson, College of William and Mary, Williamsburg, Virginia

C. F. Voliva, Pharmacia Corporation, Chesterfield, Missouri

Jianli Wang, Virginia Polytechnic Institute and State University, Blacksburg, Virginia

William P. Weber, University of Southern California, Los Angeles, California

Alan Wertsching, Idaho National Engineering and Environmental Laboratory, Idaho Falls, Idaho

Ozlem Yavuz, University of Central Florida, Orlando, Florida

Torsten Zerfaß, Albert-Ludwigs Universität, Freiburg, Germany

Shiying Zheng, Eastman Kodak, Rochester New York

J. Zobell, Pharmacia Corporation, Chesterfield, Missouri 


\section{Preface}

Most synthetic and natural polymers can be divided according to whether they are condensation or vinyl polymers. While much publicity has focused on funtionalized vinyl polymers, little has been done to bring together material dealing with functionalized condensation polymers. Yet, functionalized condensation polymers form an ever increasingly important, but diverse, group of materials that are important in our search for new materials for the 21 st century. They form a major part of the important basis for the new and explosive nanotechnology, drug delivery systems, specific multisite catalysts, communication technology, etc.

For synthetic polymers, on a bulk basis, vinyl polymers are present in about a two to three times basis. By comparison, in nature, the vast majority of polymers are of the condensation variety.

Functionalized or functional condensation polymers are condensation polymers that contain functional groups that are either present prior to polymer formation, introduced during polymerization, or introduced subsequent to the formation of the polymer. The polymers can be linear, branched, hyper-branched, dendritic, etc. They are important reagents in the formation of ordered polymer assemblies and new architectural dendritic-like materials.

Condensation polymers offer advantages not offered by vinyl polymers including offering different kinds of binding sites; the potential for easy biodegradability; offering different reactivities undergoing reaction with different reagents under different reaction conditions; offering better tailoring of end-products; offering different tendencies (such as fiber formation); and offering different physical and chemical properties.

This book is based, in part, on an international symposium given in April 2001 as part of the national American Chemical Society meeting in San Diego, California, which was sponsored by the Division of Polymeric Materials: Science and Engineering. About forty presentations were made at the meeting.

Sample areas emphasized included dendrimers, control release of drugs, nanostructural materials, controlled biomedical recognition, and controllable electrolyte and electrical properties.

Of these presentations, about half were chosen to be included in this volume. Areas chosen for this book are those where functional condensation polymers play an especially critical role. These are nanomaterials, light and energy, bioactivity and biomaterials, and enhanced physical properties. 
The book is not comprehensive, but illustrative, with the authors selected to reflect the broadness and wealth of materials that are functional condensation polymers in the areas chosen for emphasis in this book. The authors were encouraged to place their particular contribution in perspective and to make predictions of where their particular area is going. 


\section{Contents}

\section{A. Nano Materials}

\section{Lanthanide (III) Oxide Nanocomposites with}

Hexafluoroisopropylidine-Based Polyimides

D. Scott Thompson, D. W. Thompson, Robin E. Southward

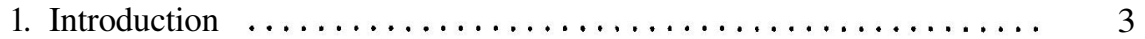

1.1 Hexafluoroisopropylidene-containing polyimides $\ldots \ldots \ldots \ldots . . \ldots 3$

1.2 Potential applications of fluorinated polyimides $\ldots \ldots \ldots \ldots . . \ldots$

1.3 Oxo-metal-polyimide composites $\ldots \ldots \ldots \ldots \ldots \ldots \ldots \ldots \ldots . \ldots \ldots$

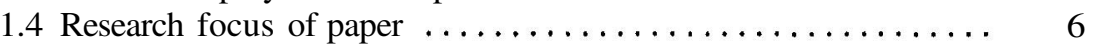

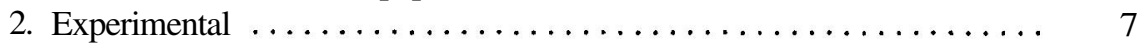

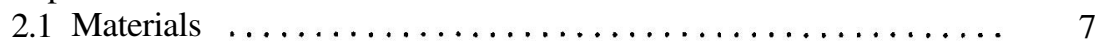

2.2 Preparation of diquotris(2,4-pentanedionato)-lanthanum(III) and diaquotris(2,4-pentadionato)gadolinium(III) monohydrate .... 7

2.3 Preparation of polyimides $\ldots \ldots \ldots \ldots \ldots \ldots \ldots \ldots \ldots . \ldots \ldots$

2.4 Preparation and characterization of oxo-lanthanum-polyimide

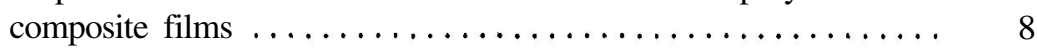

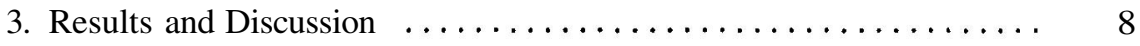

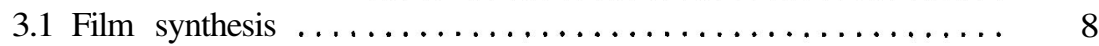

3.2 Film properties: linear coefficients of thermal expansion and thermal and mechanical properties $\ldots \ldots \ldots \ldots \ldots \ldots \ldots .9$

3.3 Rationale for the use of lanthanide(III)-based inorganic phases . . 13

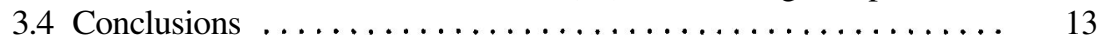

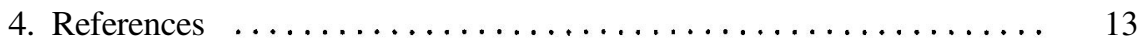

\section{Fumaryl Chloride and Maleic Anhydride Derived Crosslinked}

Functional Polymers and Nano Structures $\ldots \ldots \ldots \ldots \ldots \ldots$

Sam-Shajing Sun, Shahin Maaref, Carl E. Bonner

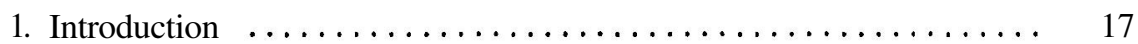

1.1 Need for functional polymer nano structures $\ldots \ldots \ldots \ldots \ldots \ldots . \ldots \ldots$

1.2 Polymer NLO waveguide $\ldots \ldots \ldots \ldots \ldots \ldots \ldots \ldots \ldots \ldots . \ldots \ldots$

2. A Brief Survey of Crosslinked NLO Polymers $\ldots \ldots \ldots \ldots \ldots \ldots 22$

2.1 Thermally crosslinked systems $\ldots \ldots \ldots \ldots \ldots \ldots \ldots \ldots \ldots . \ldots \ldots$ 
2.2 Photo crosslinked systems $\ldots \ldots \ldots \ldots \ldots \ldots \ldots \ldots \ldots \ldots . \ldots \ldots$

3. Fumaryl Chloride and Maleic Anhydride Derived Crosslinked NLO

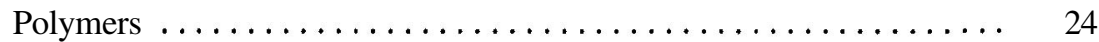

3.1 Fumarate type crosslinkable polymers $\ldots \ldots \ldots \ldots \ldots \ldots \ldots 24$

3.2 NLO Polymers from fumarate type crosslinked polymers ..... 26

4. Summary and Future Research $\ldots \ldots \ldots \ldots \ldots \ldots \ldots \ldots . \ldots . \ldots 28$

5. References and Notes ........................ 29

3. Humeral Immune Response to Polymeric Nanomaterials . . . . . . . 31

Stephen C. Lee, R. Parthasarathy, K. Botwin, D. Kunneman, E. Rowold, G. Lange, J. Zobell, T. Beck, T. Miller, R. Jansson, C. F. Voliva

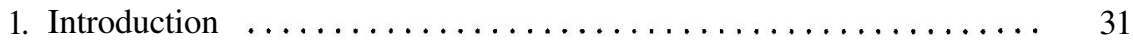

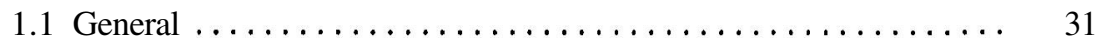

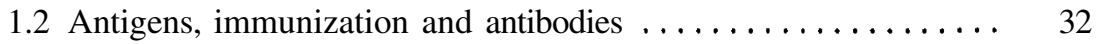

1.3 Current studies .............................. 35

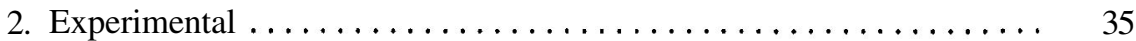

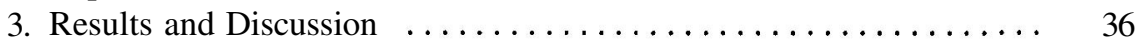

3.1 Immune responses to PAMMAN dendrimers $\ldots \ldots \ldots \ldots \ldots \ldots . \ldots . \ldots$

3.2 Antibody recognition to PAMAN dendrimers $\ldots \ldots \ldots \ldots \ldots . . . . . \ldots$

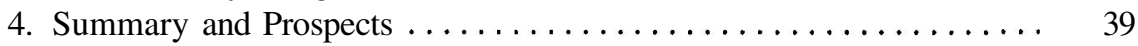

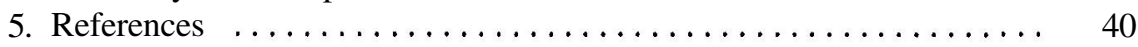

\section{Preparation and Characterization of Novel Polymer/Silicate}

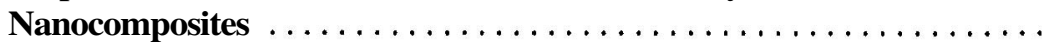

Mason K. Harrup, Alan K. Wertsching, Michael G. Jones

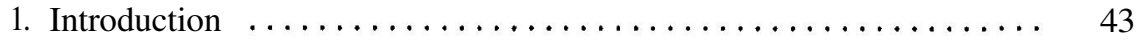

1.1 Nanocomposite classification system $\ldots \ldots \ldots \ldots \ldots \ldots \ldots \ldots, 43$

2. Experimental ........................... 46

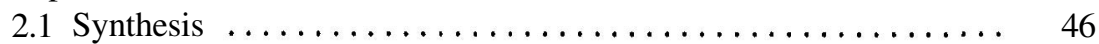

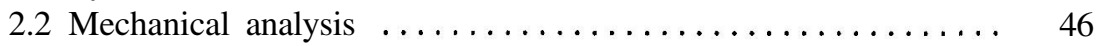

2.3 ESEM Measurements ........................ 47

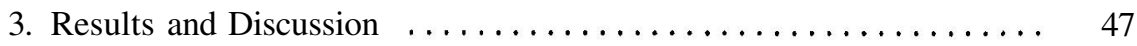

3.1 Polyphosphazone nanocomposites $\ldots \ldots \ldots \ldots \ldots \ldots \ldots \ldots, 47$

3.2 Organic polymer nanocomposites $\ldots \ldots \ldots \ldots \ldots \ldots \ldots \ldots \ldots, 50$

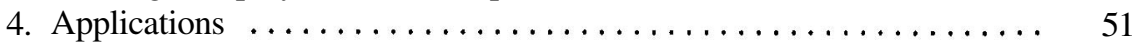

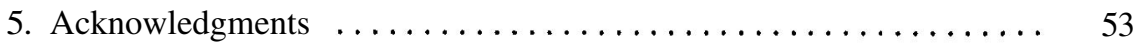

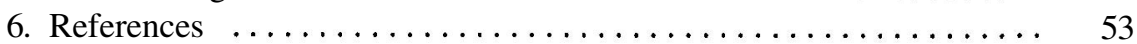


5. Metallocene Hematoporphyrins as Analytical Reagents-Nickel (II) Metal Adsorption Studies of Group IVB Metallocene Polymers Derived from Hematoporphyrin IX ................ 55

Charles E. Carraher, Jr., Jerome E. Haky, Alberto Rivalta

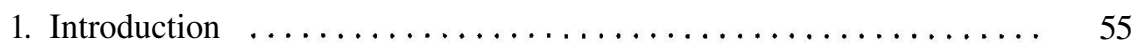

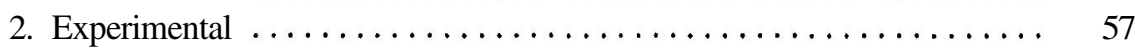

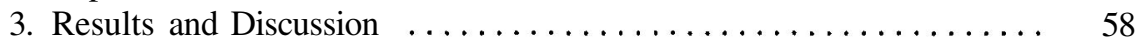

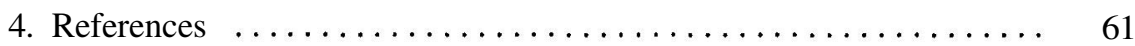

6. Polyester Ionomers as Functional Compatibilizers for Blends with Condensation Polymers and Nanocomposites $\ldots \ldots \ldots \ldots \ldots$

Robert B. Moore, Timothy L. Boykin, and Grant D. Barber

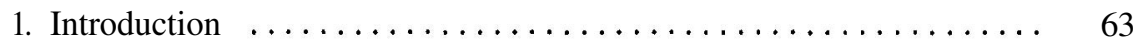

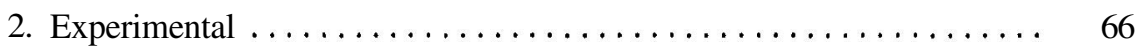

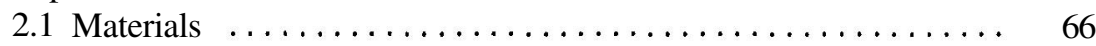

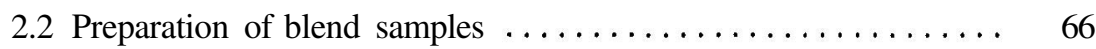

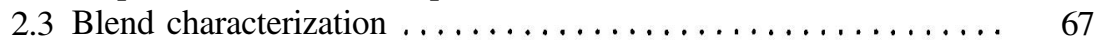

2.4 Preparation of nanocomposite samples $\ldots \ldots \ldots \ldots \ldots \ldots \ldots 68$

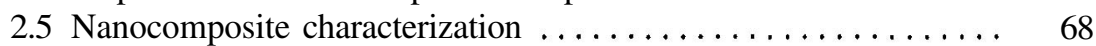

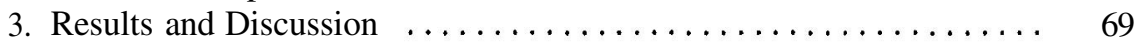

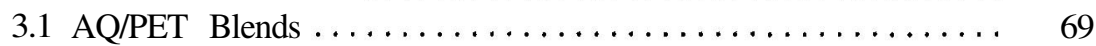

3.2 AQ/N66 Blends $\ldots \ldots \ldots \ldots \ldots \ldots \ldots \ldots \ldots \ldots \ldots \ldots \ldots . \ldots \ldots$

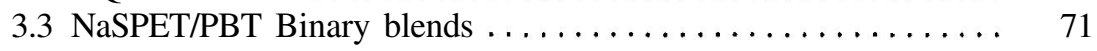

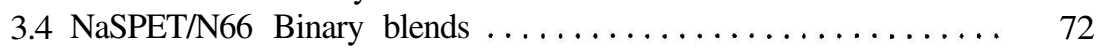

3.5 NaSPET/PET/N66 Compatibilized blends . ........... 73

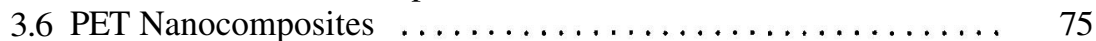

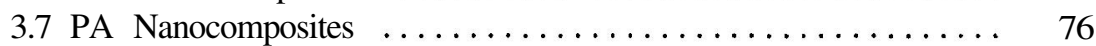

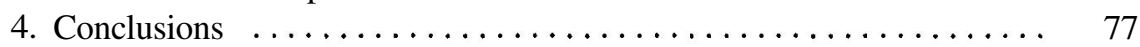

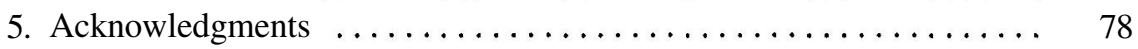

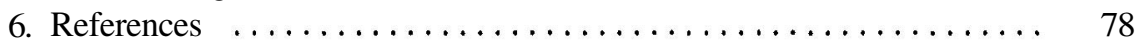

B. Light and Energy

7. Sulfonated and Carboxylated Copoly(Arylenesulfone)s for Fuel

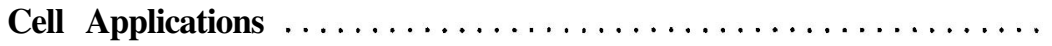

Dirk Poppe, Torsten Zerfaß, Rolf Mulhaupt, Holger Frey

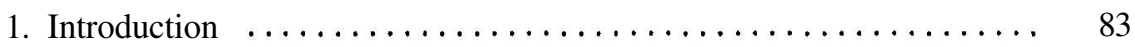

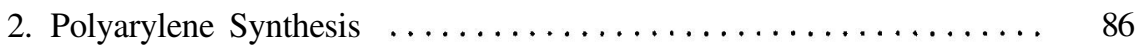


2.1 Polyarylenesulfones with $\mathrm{SO}_{3} \mathrm{H}$ groups $\ldots \ldots \ldots \ldots \ldots \ldots \ldots . \ldots 87$

2.2 Polyarylenes with $\mathrm{COOH}$ groups $\ldots \ldots \ldots \ldots \ldots \ldots \ldots \ldots \ldots . \ldots \ldots$

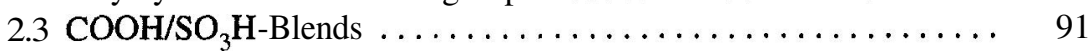

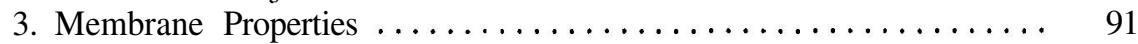

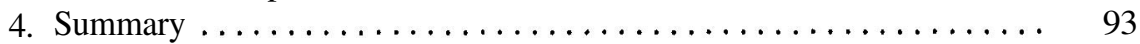

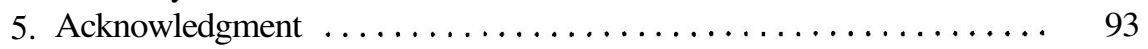

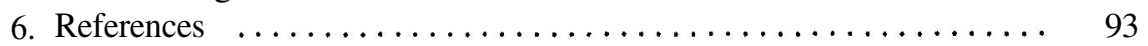

8. Preparation and Properties of Sulfonated or Phosphonated Polybenzimidazoles and Polybenzoxazoles

Yoshimitsu Sakaguchi, Kota Kitamura, Junko Nakao, Shiro Hamamoto, Hiroshi Tochimori, Satoshi Takase

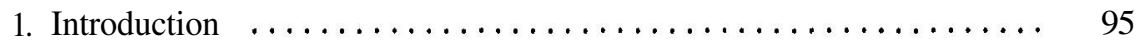

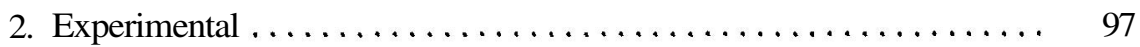

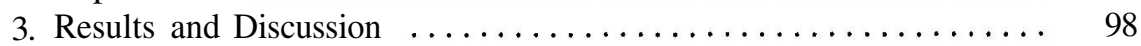

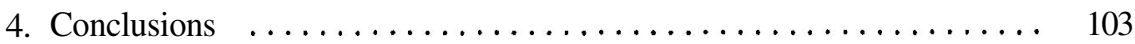

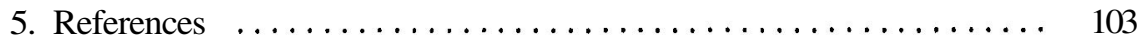

9. Design of Conjugated Polymers for Single Layer Light Emitting Diodes ......................................... 105

Zhonghua Peng

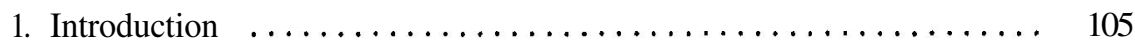

2. Conjugated Polymers Exhibiting High Solid State PL Efficiencies .. 106

3. Exploring Approaches Toward Balanced Charge Injection and Transport .................................. 109

4. Polymers with Both High PL Efficiency and Balanced ChargeInjection Properties .......................... 116

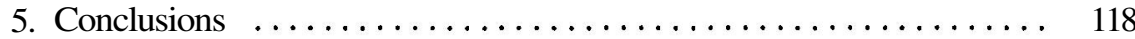

6. References ................................ 119

10. Synthesis and Characterization of Novel Blue Light-Emitting Polymers Containing Dinaphthylanthracene

Shiying Zheng, Jianmin Shi

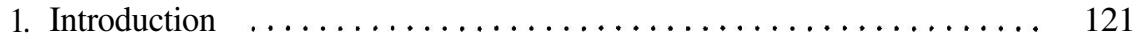

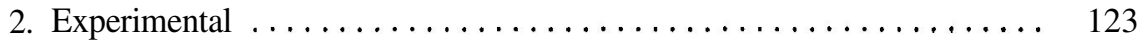

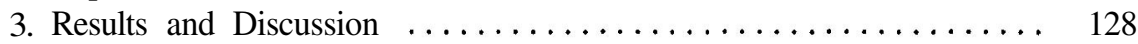

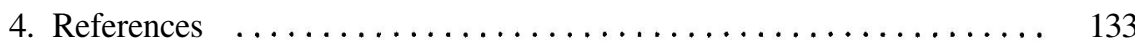


11. Novel Two-Photon Absorbing Polymers . . . . . . . . . . . . . 135

Kevin D. Belfield, Alma R. Morales, Stephen Andrasik,

Katherine J. Schaefer, Ozlem Yavuz, Victor M. Chapela, Judith Percino

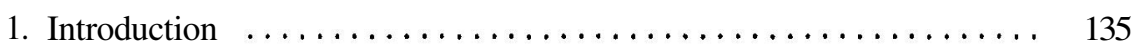

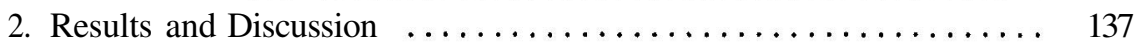

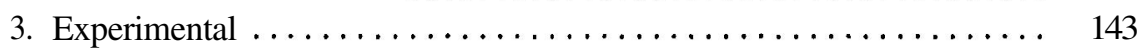

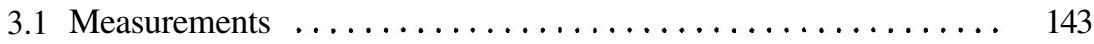

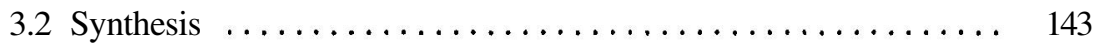

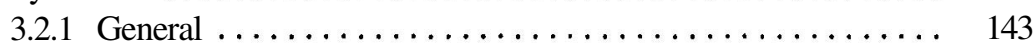

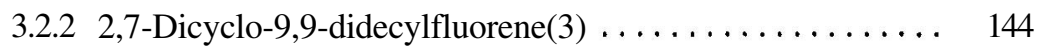

3.2.3 Poly(benzo[1,2-d:4,5-d' ]bisthiazole-9,9-didecylfluorene)(5) 144

3.2.4 7-Benzothiazol-2-yl-9,9-didecylfluoren-2-ylamine-modified

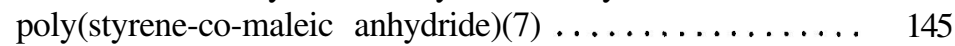

3.2.5 7-Benzothiazol-2-yl-9,9-didecylfluorene-2-ylaminemodified poly(ethylene-g-maleic anhydride)(8) ....... 146

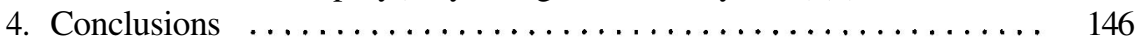

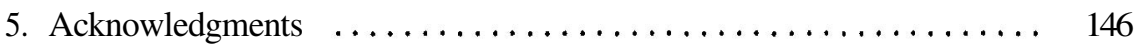

6. References $\ldots \ldots \ldots \ldots \ldots \ldots \ldots \ldots \ldots \ldots \ldots \ldots \ldots \ldots . \ldots \ldots$

\section{Bioactivity and Biomaterials}

12. Natural Functional Condensation Polymer Feedstocks .......... 151

Charles E. Carraher, Jr.

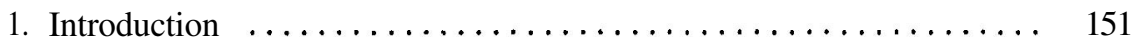

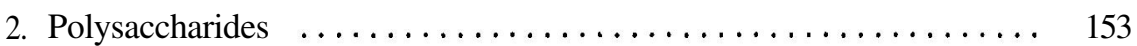

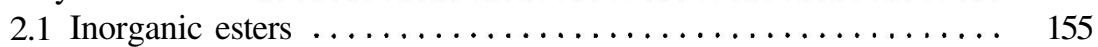

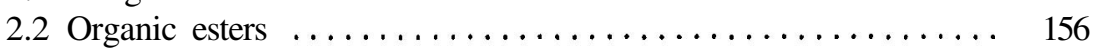

2.3 Other polysaccharides $\ldots \ldots \ldots \ldots \ldots \ldots \ldots \ldots \ldots \ldots \ldots \ldots, 160$

2.3.1 Homopolysaccharides $\ldots \ldots \ldots \ldots \ldots \ldots \ldots \ldots \ldots \ldots . \ldots \ldots$

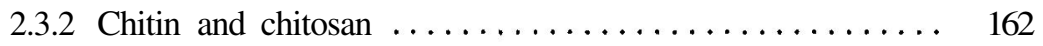

2.5 Heteropolysaccharides . . . . . . . . . . . . . . . . . . 165

3. Nucleic Acids .............................. 169

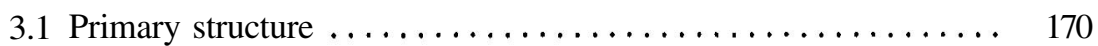

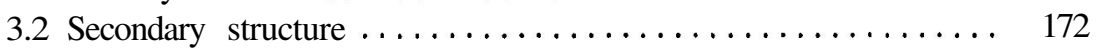

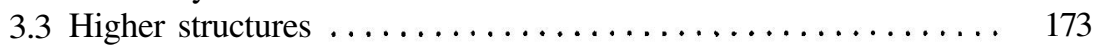

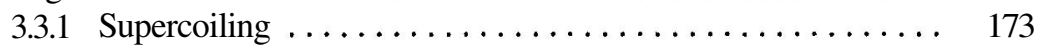

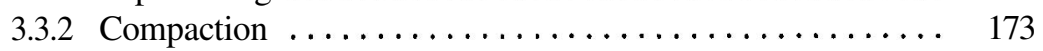

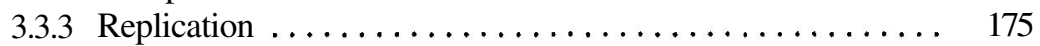

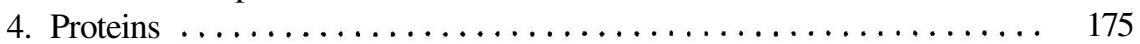

4.1 General structures $\ldots \ldots \ldots \ldots \ldots \ldots \ldots \ldots \ldots \ldots \ldots \ldots \ldots$

4.2 Secondary structure $\ldots \ldots \ldots \ldots \ldots \ldots \ldots \ldots \ldots \ldots \ldots \ldots$ 
4.3 Keratines .............................. 177

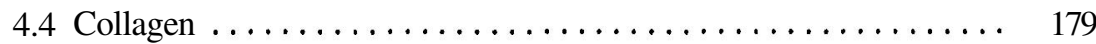

4.5 Tertiary structure $\ldots \ldots \ldots \ldots \ldots \ldots \ldots \ldots \ldots \ldots \ldots \ldots \ldots . \ldots \ldots$

4.6 Globular proteins $\ldots \ldots \ldots \ldots \ldots \ldots \ldots \ldots \ldots \ldots \ldots \ldots \ldots \ldots \ldots$

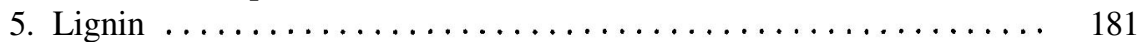

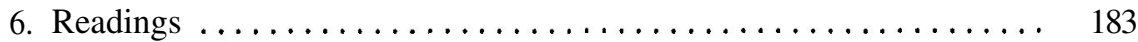

13. Functional Polymers Derived from Condensation of Itaconic Andydride with Poly(E-Caprolactone)diol and with

Poly(Ethylene glycol) . . . . . . . . . . . . . . . . . . . . . 185

Monica Ramos and Samuel J. Huang

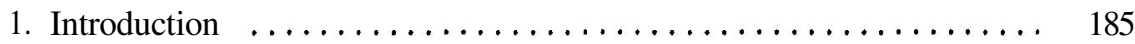

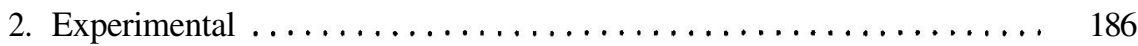

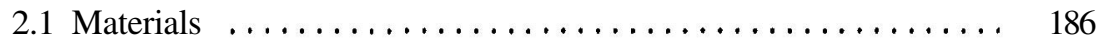

2.2 Instrumentation $\ldots \ldots \ldots \ldots \ldots \ldots \ldots \ldots \ldots \ldots \ldots \ldots \ldots, 187$

2.3 Synthesis of polycaprolactone diitaconates $\ldots \ldots \ldots \ldots \ldots \ldots 187$

2.4 Synthesis of poly(ethylene glycol) diitaconates . . . . . . . . 187

2.5 Crosslinkning procedure $\ldots \ldots \ldots \ldots \ldots \ldots \ldots \ldots \ldots \ldots \ldots \ldots$

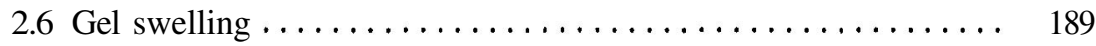

3. Results and Discussion $\ldots \ldots \ldots \ldots \ldots \ldots \ldots \ldots \ldots \ldots \ldots . \ldots \ldots$

3.1 Spectral characteristics of PCLDIs and PEGDIs macromonomers 189

3.2 Characterization of the hydrogels $\ldots \ldots \ldots \ldots \ldots \ldots \ldots \ldots \ldots . \ldots 19$

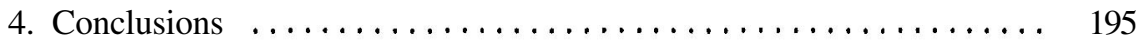

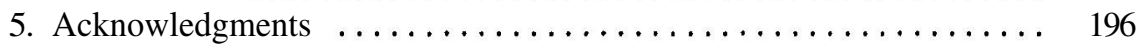

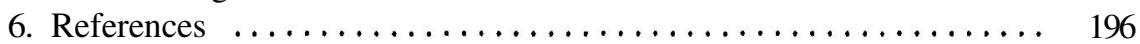

14. Organometallic Condensation Polymers as Anticancer Drugs ..... 199

Deborah W. Siegmann-Louda, Charles E. Carraher, Jr., Fred Pflueger, David Nagy, John R. Ross

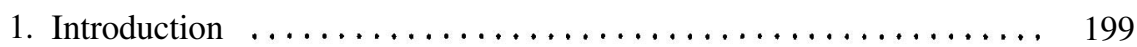

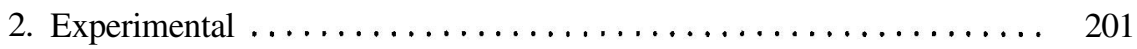

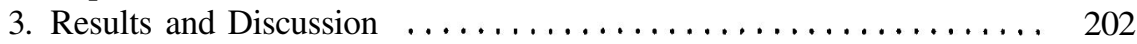

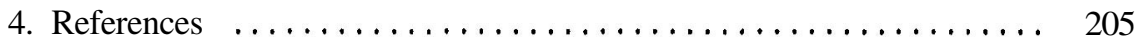

15. Synthesis and Structural Characterization of Chelation Products Between Chitosan and Tetrachloroplatinate Towards the Synthesis of Water Soluble Cancer Drugs

Charles E. Carraher, Jr., Ann-Marie Francis,

Deborah W. Siegmann-Louda

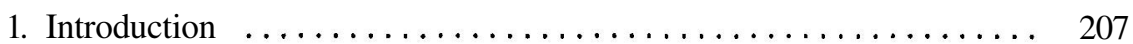

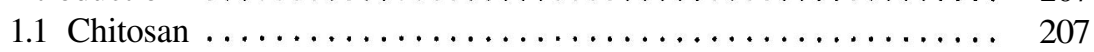




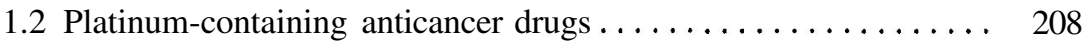

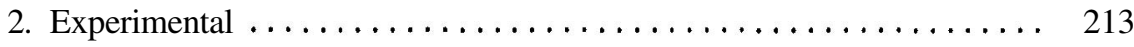

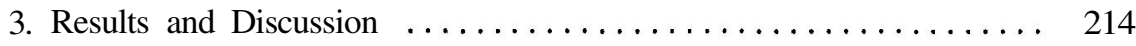

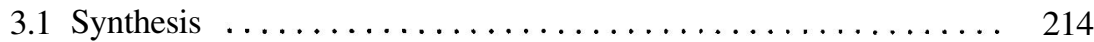

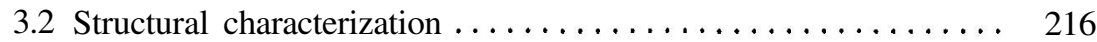

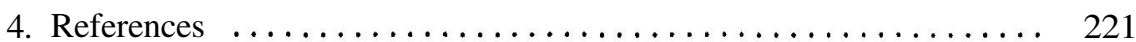

16. Condensation Polymers as Controlled Release Materials for Enhanced Plant and Food Production: Influences of Gibberellic Acid and Gibberellic Acid-Containing Polymers on Food Crop Production

Charles E. Carraher, Jr., Herbert Stewart, Shawn M. Carraher, Donna M. Chamely, Wesley W. Learned, James Helmy, Kumudi Abey, Alicia R. Salamone

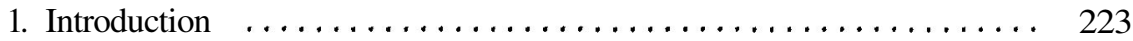

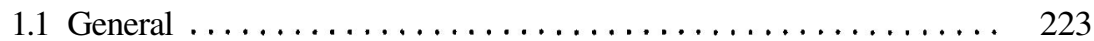

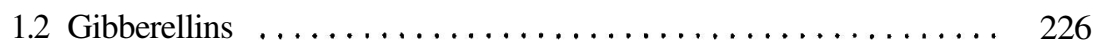

1.3 Auxins ................................. 228

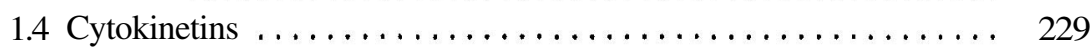

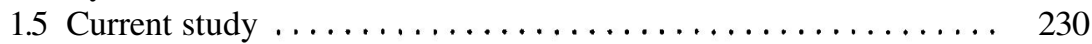

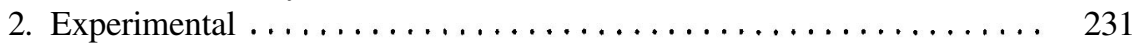

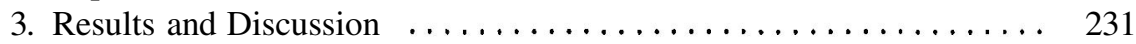

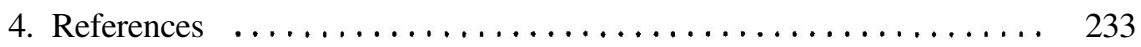

\section{Enhanced Physical Properties}

\section{2,6-Anthracenedicarboxylate-Containing Polyesters and}

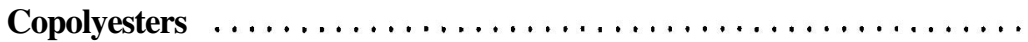

David M. Collard, David A. Schiraldi

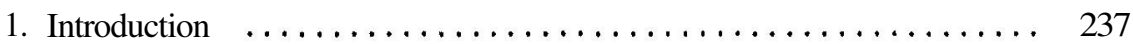

2. Monomer Synthesis and Polymerization $\ldots \ldots \ldots \ldots \ldots \ldots \ldots . \ldots \ldots$

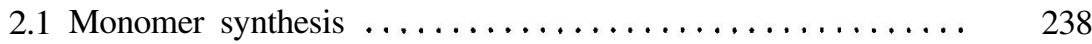

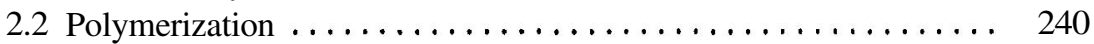

3. Poly(alkylene anthracene 2,6-dicarboxylate)s, PnA . . . . . . . . . 241

4. Poly(ethylene 2,6-anthracenedicarboxylate-co-terephthalate)s, PET-A 242

5. Diels-Alder Crosslinking and Grafting Reactions of PET-A . . . . . . 242

6. Photocrosslinking of PET-A . . . . . . . . . . . . . . . . . . . . 243

7. Chain Extension of Anthracene-terminated PET $\ldots \ldots \ldots \ldots \ldots 245$

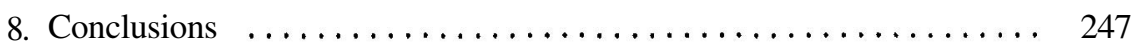

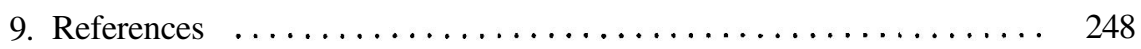


18. Synthesis and Characterization of Ionic and Non-ionic Terminated Amorphous Poly(Ethylene isophthalate)

Huaiying Kang, R. Scott Armentrout, Jianli Wang, Timothy E. Long

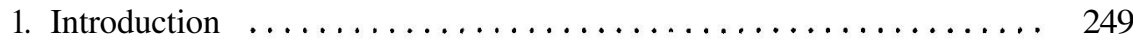

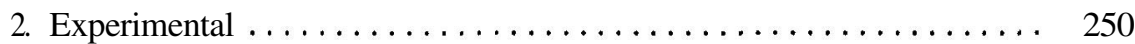

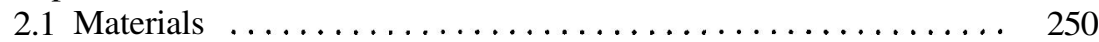

2.2 Preparation of catalyst solutions .................. 251

2.3 Synthesis of non-terminated high molecular weight poly(ethylene isophthalate) (PEI) . ............... 251

2.4 Synthesis of sulfonate terminated PEI ionomers (PEI-SSBA) . . 251

2.5 Synthesis of dodeconol terminated poly(ethylene isophthalate)

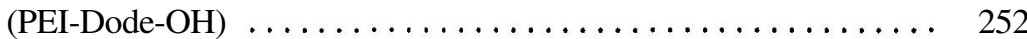

2.6 Polymer characterization . . . . . . . . . . . . . . . . . . . . 253

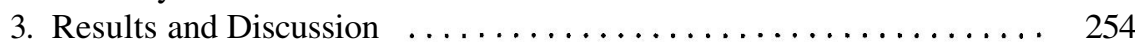

3.1 GPC and NMR Analysis . . . . . . . . . . . . . . 254

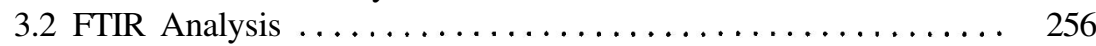

3.3 DSC and TGA Analysis $\ldots \ldots \ldots \ldots \ldots \ldots \ldots \ldots \ldots \ldots . \ldots \ldots \ldots$

3.4 Solution viscometry study $\ldots \ldots \ldots \ldots \ldots \ldots \ldots \ldots \ldots \ldots \ldots \ldots \ldots$

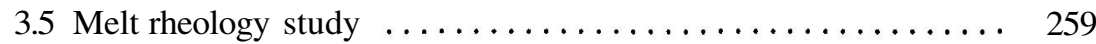

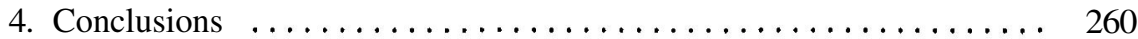

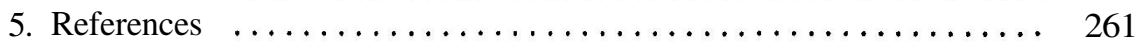

19. Synthesis, Characterization and Application of Functional Condensation Polymers from Anhydride Modified Polystyrene and Their Sulfonic Acid Resins

Sakuntala Chatterjee Ganguly

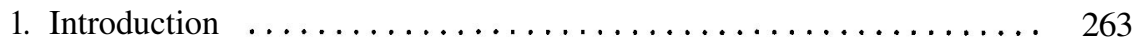

1.1 Functional condensation polymer ................ 263

1.1.1 Synthesis and chemical modification of a polymer in bulk 263

1.1.2 Surface modification of a polymer by chemical modification ....................... 266

1.1.3 Surface modification of a polymer by interpenetrating

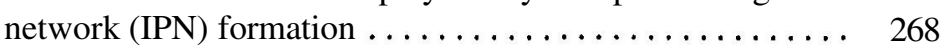

2. Experimental $\ldots \ldots \ldots \ldots \ldots \ldots \ldots \ldots \ldots \ldots \ldots \ldots \ldots \ldots \ldots . \ldots \ldots$

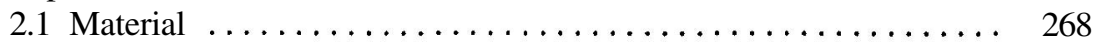

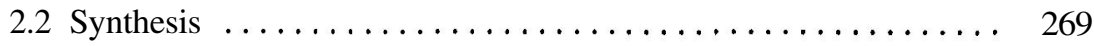

2.2.1 Preparation of PSPA, PSTM, PSTHPA, PSPMDA . . . . 269

2.2.2 Synthesis of sulfonic acid resins PSPAS, PSTMAS,

PSTHPAS, PSPMDAS and their sodium salts .......... 269

2.2.3 Preparation of PSNTDA, PSPTDA and PS6FDA ...... 269

2.2.4 Preparation of PSPMDA film ............. 270 
2.2.5 Preparation of dianhydride coated Teflon membrane .... 270

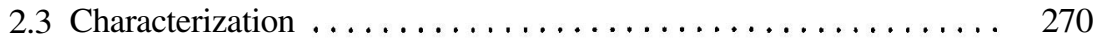

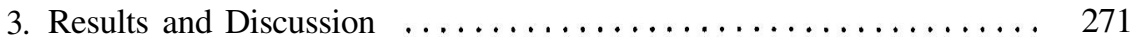

3.1 Characterization of PSPA, PSTMA, PSTHPA, PSPMDA ..... 271

3.2 Novel membrane from pyromellitic dianhydride modified polystyrene with controlled pore size on micro- and macrolevels 276

3.3 Structural characterization of sulfonic acids resins PSPAS, PSTMAS, PSTHPAS and PSPMDAS

3.4 Structural characterization of PSPTDA, PS6FDA and PSNTDA

3.5 Low voltage scanning electron microscopy of a surface modified K-100 teflon membrane and thermal analysis studies of several anhydride modified Nafioin 417 membranes ............. 283

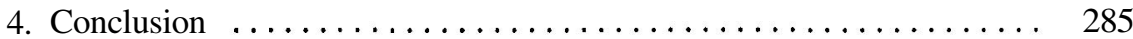

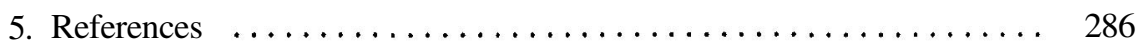

20. Condensation Copolymerization via Ru-Catalyzed Reaction of o-Quinones or $\alpha$-Diketones with $\alpha, \sigma$-Dihydridooligodimethylsiloxanes

Joseph M. Mabry, William P. Weber

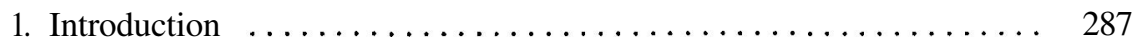

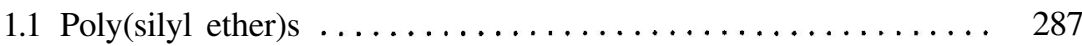

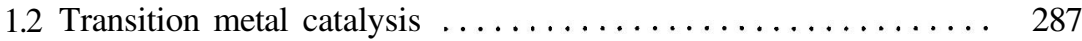

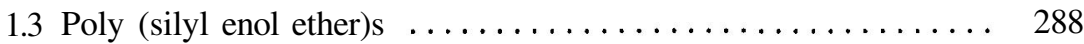

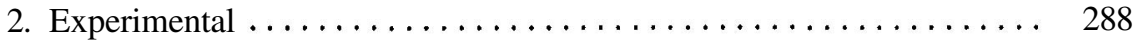

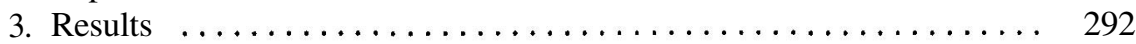

3.1 Results . . . . . . . . . . . . . . . . . . . . . . . . . . . . . . 292

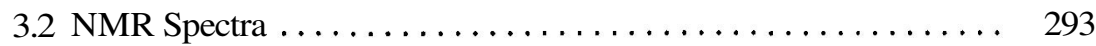

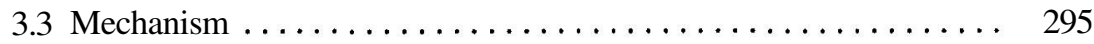

3.4 Luminescence ............................ 296

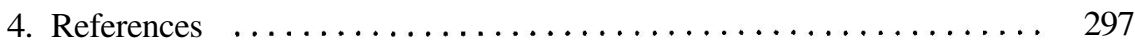

21. Gel-Drawn Poly(p-phenylenepyromellitimide) $\ldots \ldots \ldots \ldots . \ldots \ldots 299$ Jim Sadanobu, Rei Nishio

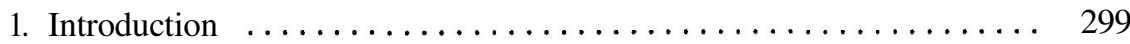

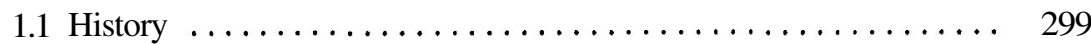

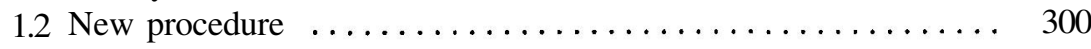

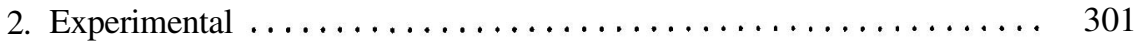

2.1 Preparation of polyamic acid solution $\ldots \ldots \ldots \ldots \ldots \ldots \ldots, 301$

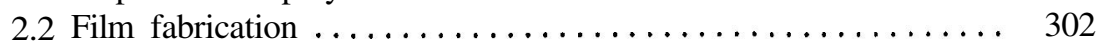

2.3 Characterization of polymer $\ldots \ldots \ldots \ldots \ldots \ldots \ldots \ldots \ldots \ldots \ldots$ 


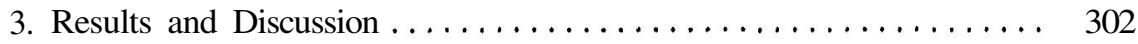

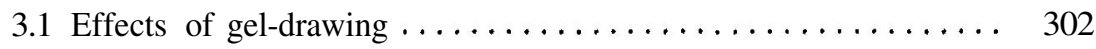

3.2 Microstructure developed in imidized film $\ldots \ldots \ldots \ldots \ldots \ldots 305$

4. Properties of PPPI film $\ldots \ldots \ldots \ldots \ldots \ldots \ldots \ldots \ldots \ldots \ldots \ldots \ldots \ldots$

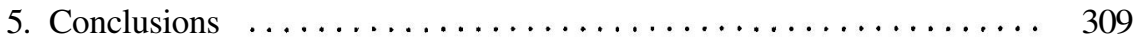

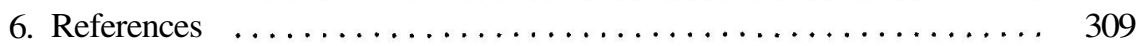

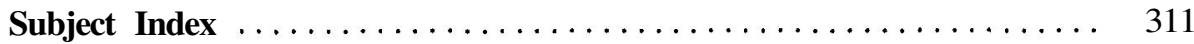

